\title{
The Density-Dependent Correlations among Observables in Nuclear Matter and Hyperon-Rich Neutron Stars
}

\author{
Schun T. Uechi ${ }^{1}$ and Hiroshi Uechi ${ }^{2}$ \\ ${ }^{1}$ Department of Physics, Osaka City University, 3-3-138 Sugimoto, Sumiyoshi-ku, Osaka 558-8585, Japan \\ ${ }^{2}$ Department of Distributions and Communication Sciences, Osaka Gakuin University, 2-36-1 Kishibe- \\ minami, Suita, Osaka 564-8511, Japan \\ Correspondence should be addressed to Hiroshi Uechi, uechi@ogu.ac.jp
}

Received 8 August 2008; Revised 11 December 2008; Accepted 19 February 2009

Recommended by Kate Scholberg

The conserving $\sigma-\omega-\rho$ mean-field approximation with nonlinear interactions of hadrons has been applied to examine properties of nuclear matter and hyperonic neutron stars. The nonlinear interactions that will produce density-dependent effective masses and coupling constants of hadrons are included in order to examine density correlations among properties of nuclear matter and neutron stars such as binding energy, incompressibility, $K$, symmetry energy, $a_{4}$, hyperon-onset density, and maximum masses of neutron stars. The conditions of conserving approximations in order to maintain thermodynamic consistency to an approximation are essential for the analysis of density-dependent correlations.

Copyright (C) 2009 S. T. Uechi and H. Uechi. This is an open access article distributed under the Creative Commons Attribution License, which permits unrestricted use, distribution, and reproduction in any medium, provided the original work is properly cited.

\section{Introduction}

The linear and nonlinear hadronic mean-field approximations have been extensively applied to finite nuclei, nuclear matter, and neutron stars [1-3]. The ground state of symmetric nuclear matter has always been a fundamental physical system in the understanding of complicated normal and high density, exotic nuclear many-body systems. The high-density matter such as a neutron star has been actively investigated, observed masses of hadronic neutron stars are above $1.3 M_{\odot}$, and the maximum masses of neutron stars are expected to be below $2.5 M_{\odot}$ $[4,5]$. The neutron stars are also speculated to be hyperon-mixed nuclear matter whose equation of state will provide us with important conditions to understand interactions of nuclear physics [6-9].

The current nonlinear mean-field approximation is constructed with $\sigma-\omega-\rho$ selfinteractions and mixing interactions of mesons. The nonlinear interactions of mesons are renormalized as the self-consistent effective masses and effective coupling constants by 
the requirement of thermodynamic consistency, or equivalently the theory of conserving approximations [10-21]. The self-consistent effective masses and effective coupling constants of mesons are essential to maintain self-consistency in nonlinear approximations and examine correlations among properties of nuclear and high-density, hyperonic matter.

The density-dependent correlations among properties of nuclear matter and neutron stars have been discussed in terms of effective masses and coupling constants of mesons and baryons, which are defined self-consistently to maintain conditions of conserving approximations $[20,21]$. The nonlinear $\sigma-\omega-\rho$ mean-field approximation suggests that density-dependent correlations induced by nonlinear interactions be significant and so, the analysis helps us understand nuclear many-body interactions.

The Lagrangian of nonlinear $\sigma-\omega-\rho$ mean-field approximation which yields densitydependent effective masses and coupling constants is [20,21]

$$
\begin{aligned}
\mathcal{L}_{\mathrm{NHA}}= & \sum_{B} \bar{\psi}_{B}\left[i \gamma_{\mu} \partial^{\mu}-g_{\omega B}^{*} \gamma_{0} V_{0}-\frac{g_{\rho B}^{*}}{2} \gamma_{0} \tau_{3} R_{0}-\left(M_{B}-g_{\sigma B}^{*} \phi_{0}\right)\right] \psi_{B} \\
& -\frac{1}{2} m_{\sigma}^{2} \phi_{0}^{2}-\frac{g_{\sigma 3}}{3 !} \phi_{0}^{3}-\frac{g_{\sigma 4}}{4 !} \phi_{0}^{4}+\frac{1}{2} m_{\omega}^{2} V_{0}^{2}+\frac{g_{\omega 4}}{4 !} V_{0}^{4}+\frac{g_{\sigma \omega}}{4} \phi_{0}^{2} V_{0}^{2} \\
& +\frac{1}{2} m_{\rho}^{2} R_{0}^{2}+\frac{g_{\rho 4}}{4 !} R_{0}^{4}+\frac{g_{\sigma \rho}}{4} \phi_{0}^{2} R_{0}^{2}+\frac{g_{\omega \rho}}{4} V_{0}^{2} R_{0}^{2}+\sum_{l} \bar{\psi}_{l}\left(i \gamma_{\mu} \partial^{\mu}-m_{l}\right) \psi_{l},
\end{aligned}
$$

where $\psi_{B}(B=n, p, \Lambda, \Sigma, \ldots)$ and $\psi_{l}\left(l=e^{-}, \mu^{-}\right)$denote the field of baryons and leptons, respectively. The meson-fields operators are replaced by expectation values in the ground state: $\phi_{0}$ for the $\sigma$-field, $V_{0}$ for the vector-isoscalar $\omega$-meson, $V_{\mu} V^{\mu}=V_{0}^{2}-\mathbf{V}^{2},(\mu=0,1,2,3)$; the neutral $\rho$-meson mean-field, $R_{0}$, is chosen for $\tau_{3}$-direction in isospin space. The masses in (1.1) are: $M=939 \mathrm{MeV}, m_{\sigma}=550 \mathrm{MeV}, m_{\omega}=783 \mathrm{MeV}$, and $m_{\rho}=770 \mathrm{MeV}$, in order to compare the effects of nonlinear interactions and hyperon-matter with those of the linear $\sigma-\omega$ approximation discussed by Serot and Walecka [1].

The nonlinear model is motivated by preserving the structure of Serot and Walecka's linear $\sigma-\omega$ mean-field approximation [1], Lorentz-invariance and renormalizability, thermodynamic consistency: Landau's hypothesis of quasiparticles [22, 23], the Hugenholtz-van Hove theorem [24], and the virial theorem [25], and conditions of conserving approximations $[11,12,20,21]$. The concept of effective masses and effective coupling constants is naturally generated by nonlinear interactions of mesons and baryons. The conditions of conserving approximations will require the functional form of single particle energy, effective masses, and coupling constants for self-consistency, and then empirical values of low-density nuclear matter and high-density neutron matter will be restricted with the effective masses and coupling constants $[20,21]$. In other words, the admissible values of effective coupling constants and masses are confined in certain values due to strong density-dependent correlations among physical quantities of nuclear matter and neutron stars. The purpose of the analysis is to study density-dependent correlations among properties of nuclear matter and neutron stars with the minimum constraints at nuclear matter saturation and the maximum masses of hyperonic neutron stars.

The properties at saturation of symmetric nuclear matter and neutron stars are taken so as to fix nonlinear coupling constants. The binding energy at saturation is fixed as $-15.75 \mathrm{MeV}$ at $k_{F}=1.30 \mathrm{fm}^{-1}$, and the symmetry energy, $a_{4}=30.0 \mathrm{MeV}$. Then, the minimum value of incompressibility, $K$, is determined by simultaneously maintaining the maximum masses of 
isospin-asymmetric neutron stars to be $M_{\max }(n, p, e)=2.50 M_{\odot}[20,21]$. In this way, the density-dependent correlations among properties of nuclear matter and hyperonic neutron stars, $M_{\text {star }}\left(n, p, \Sigma^{-}, \Lambda, e\right)$, are investigated. The constraints will confine nonlinear parameters within certain values and suppress the effect of nonlinear interactions.

It can be checked numerically that the baryons and an electron, $\left(n, p, \Sigma^{-}, \Lambda, e\right)$, are sufficient to determine the masses of hyperonic neutron stars; other hyperons can be included, but because of charge neutrality and self-consistency, the other hyperon-onset densities are pushed up to high densities where EOSs of the hyperons are not so important to determine the properties of neutron stars. Consequently, other hyperons produce small density-dependent correlations to properties of nuclear matter and neutron stars compared to $\left(n, p, \Sigma^{-}, \Lambda, e\right)$ matter. In other words, the EOS of $\left(n, p, \Sigma^{-}, \Lambda, e\right)$ matter dominates the densityregion decisive to properties of neutron stars. This is one of the important results obtained in the current conserving nonlinear mean-field approximation.

The self-consistency required by thermodynamic consistency restricts values of nonlinear coefficients. The suppressions of nonlinear coefficients and nonlinear interactions are directly observed in self-consistent effective masses and self-energies of mesons and baryons, which are discussed as naturalness of nonlinear corrections [20, 21]. The more accurately we can determine the observables and constraints for nuclear and high-density matter, the better we would be able to understand interactions and correlations, or limitations of hadronic models. The conserving mean-field approximation is applied in order to extract density-dependent correlations among properties of nuclear matter and high-density, hyperonic matter.

\section{Self-Consistent Effective Masses and Coupling Constants of Mesons}

The density-dependent, effective coupling constants are assumed to be induced by $\sigma$ field, preserving Lorentz-invariance as simple as possible. We have assumed that only nucleon-meson coupling constants are density-dependent in the current analysis since we are interested in the density correlations among properties of symmetric nuclear matter and high-density matter. The density-dependent nucleon-meson coupling constants are given by

$$
\begin{aligned}
& g_{\sigma N}^{*}=g_{\sigma N}+\left(\frac{g_{\sigma \sigma N}}{2}\right) \phi_{0}, \\
& g_{\omega N}^{*}=g_{\omega N}+g_{\sigma \omega N} \phi_{0}, \\
& \frac{g_{\rho N}^{*}}{2}=\frac{g_{\rho N}}{2}+g_{\sigma \rho N} \phi_{0} .
\end{aligned}
$$

The effective masses compatible with the effective coupling constants (2.1) are required to be

$$
\begin{aligned}
& m_{\sigma}^{* 2}=m_{\sigma}^{2}\left(1+\frac{g_{\sigma 3}}{2 m_{\sigma}^{2}} \phi_{0}+\frac{g_{\sigma 4}}{3 ! m_{\sigma}^{2}} \phi_{0}^{2}-\frac{g_{\sigma \omega}}{2 m_{\sigma}^{2}} V_{0}^{2}-\frac{g_{\sigma \rho}}{2 m_{\sigma}^{2}} R_{0}^{2}-\frac{g_{\sigma \sigma N}}{2 m_{\sigma}^{2}} \rho_{s N}\right), \\
& m_{\omega}^{* 2}=m_{\omega}^{2}\left(1+\frac{g_{\omega 4}}{3 ! m_{\omega}^{2}} V_{0}^{2}+\frac{g_{\sigma \omega}}{2 m_{\omega}^{2}} \phi_{0}^{2}+\frac{g_{\omega \rho}}{2 m_{\omega}^{2}} R_{0}^{2}\right) \\
& m_{\rho}^{* 2}=m_{\rho}^{2}\left(1+\frac{g_{\rho 4}}{3 ! m_{\rho}^{2}} R_{0}^{2}+\frac{g_{\sigma \rho}}{2 m_{\rho}^{2}} \phi_{0}^{2}+\frac{g_{\omega \rho}}{2 m_{\rho}^{2}} V_{0}^{2}\right) .
\end{aligned}
$$


The effective masses of mesons and coupling constants have to be determined selfconsistently. Note that the effective mass depends on the $(n, p)$ scalar source of nucleons, $\rho_{s N}$. The nonlinear mean-field approximation is thermodynamically consistent only if effective masses of mesons and coupling constants are renormalized as (2.1) and (2.2).

The introduction of nonlinear $\sigma \sigma N$-vertex interaction is equivalent to define the effective mass of nucleon as

$$
M_{N}^{*}=M_{N}-g_{\sigma N}^{*} \phi_{0}=M_{N}-g_{\sigma N} \phi_{0}-\left(\frac{g_{\sigma \sigma N}}{2}\right) \phi_{0}^{2}
$$

and the effective mass of hyperon $H$ is

$$
M_{H}^{*}=M_{H}-g_{\sigma H} \phi_{0}
$$

The effective masses of nucleons and hyperons are obtained from (2.3) and (2.4):

$$
M_{H}-M_{H}^{*}=\frac{g_{\sigma H}}{g_{\sigma N}^{*}}\left(M_{N}-M_{N}^{*}\right)
$$

The scalar sources of nucleons $(N)$ and hyperons $(H)$ are respectively given by [17]

$$
\Sigma_{N}^{s}=i \frac{g_{\sigma N}^{*}}{m_{\sigma}^{* 2}} \int \frac{d^{4} q}{(2 \pi)^{4}}=\operatorname{Tr}\left\{\left(g_{\sigma N}^{*}-g_{\sigma \omega N} V_{0} r^{0}-g_{\sigma \rho N} R_{0} \gamma^{0} \tau_{3}\right) G_{D}(q)\right\}=-\frac{g_{\sigma N}^{* 2}}{m_{\sigma}^{* 2}} \rho_{s N^{\prime}}^{*}
$$

where $\rho_{s}^{*}$ is the modified scalar density defined by $g_{\sigma N}^{*} \rho_{s}^{*}=g_{\sigma N}^{*} \rho_{s N}-g_{\sigma \omega N} V_{0} \rho_{B}-g_{\sigma \rho N} R_{0} \rho_{3}$. The hyperon sources are

$$
\Sigma_{H}^{S}=-\frac{g_{\sigma N}^{*}}{m_{\sigma}^{* 2}} \sum_{H} \frac{g_{\sigma H} / g_{\sigma N}^{*}}{\pi^{2}} \int_{0}^{k_{F_{H}}} d q q^{2} \frac{M_{H}^{*}}{E_{H}^{*}(q)} .
$$

where $k_{F_{B}}$ is the Fermi-momentum of the hyperon $H$, and $E_{H}^{*}(k)=\left(k^{2}+M_{H}^{* 2}\right)^{1 / 2}$. The sum is performed to baryons, and $N$ is used to denote proton and neutron: $N=(p, n)$; the hyperons are denoted as, $H=\Lambda, \Sigma^{-}, \Sigma^{0}, \Sigma^{+}, \ldots$ Although the hyperon coupling constants are not density-dependent in the current investigation, the density-dependent interactions of nucleons and self-consistency will effectively modify hyperon coupling constants as $g_{\sigma H} / g_{\sigma N}^{*}$. The density dependence of nucleon coupling constants and correlations are mainly investigated, since it is important to distinguish the density-dependences of nucleon coupling constants from those of hyperons for quantitative analyses of nuclear matter. The densitydependent interactions of hyperon coupling constants will be examined quantitatively in the near future.

The scalar sources of baryons are, respectively, given by

$$
\rho_{s B}=\sum_{B} \frac{g_{\sigma B} / g_{\sigma N}^{*}}{\pi^{2}} \int_{0}^{k_{F_{B}}} d q q^{2} \frac{M_{B}^{*}}{E_{B}^{*}(q)}
$$


where $g_{\sigma B} / g_{\sigma N}^{*} \equiv 1$ with $B=(n, p)$. The $\omega$-meson and $\rho$-meson contributions to the selfenergy are given by

$$
\Sigma_{\omega}^{\mu}=-\frac{g_{\omega N}^{* 2}}{m_{\omega}^{* 2}} \rho_{\omega} \delta_{\mu, 0}, \quad \Sigma_{\rho(n)}^{\mu}=\mp \frac{g_{\rho N}^{* 2}}{4 m_{\rho}^{* 2}} \rho_{3} \delta_{\mu, 0}
$$

where the isoscalar density, $\rho_{\omega}$, is given by

$$
\rho_{\omega}=\rho_{p}+\rho_{n}+\sum_{H} r_{H N}^{\omega} \rho_{H}
$$

and the density-dependent ratios of hyperon-nucleon coupling constants on $\omega, r_{H N^{\prime}}^{\omega}$ are defined self-consistently that will be explained in the next section. The self-energies, $\Sigma_{\rho p}^{\mu}$ and $\Sigma_{\rho n}^{\mu}$, are briefly denoted as $\left.\Sigma_{\rho(n)}^{\mu}\right)$; the isovector density is denoted as $\rho_{3}=\left(k_{F_{p}}^{3}-k_{F_{n}}^{3}\right) / 3 \pi^{2}$ where the Fermi momentum $k_{F_{p}}$ is for proton and $k_{F_{n}}$ for neutron. The baryon-isovector density, $\rho_{3 B}$, and the ratios of hyperon-nucleon coupling constants on $\rho$-meson are also defined, for example, $\rho_{3 B}=\rho_{3}+\left(g_{\rho \Sigma} / g_{\rho N}^{*}\right) \rho_{3 \Sigma}$ and $\rho_{3 \Sigma}=\rho_{\Sigma^{+}}-\rho_{\Sigma^{-}}$.

The energy density, pressure of isospin-asymmetric, and charge-neutral nuclear matter are calculated by way of the energy-momentum tensor as

$$
\begin{aligned}
\mathcal{E}_{\mathrm{NHA}}= & \sum_{B} \frac{1}{\pi^{2}} \int_{0}^{k_{F_{B}}} d k k^{2} E_{B}(k)+\frac{m_{\sigma}^{2}}{2} \phi_{0}^{2}+\frac{g_{\sigma 3}}{3 !} \phi_{0}^{3}+\frac{g_{\sigma 4}}{4 !} \phi_{0}^{4}-\frac{m_{\omega}^{2}}{2} V_{0}^{2}-\frac{g_{\omega 4}}{4 !} V_{0}^{4}-\frac{g_{\sigma \omega}}{4} \phi_{0}^{2} V_{0}^{2} \\
& -\left(\frac{m_{\rho}^{2}}{2}+\frac{g_{\rho 4}}{4 !} R_{0}^{2}+\frac{g_{\sigma \rho}}{4} \phi_{0}^{2}+\frac{g_{\omega \rho}}{4} V_{0}^{2}\right) R_{0}^{2}+\sum_{l=e^{-}, \mu^{-}} \frac{1}{\pi^{2}} \int_{0}^{k_{F_{l}}} d k k^{2} E_{l}(k), \\
p_{\mathrm{NHA}}= & \frac{1}{3 \pi^{2}} \sum_{B} \int_{0}^{k_{F_{B}}} d k \frac{k^{4}}{E_{B}^{*}(k)}-\frac{m_{\sigma}^{2}}{2} \phi_{0}^{2}-\frac{g_{\sigma 3}}{3 !} \phi_{0}^{3}-\frac{g_{\sigma 4}}{4 !} \phi_{0}^{4}+\frac{m_{\omega}^{2}}{2} V_{0}^{2}+\frac{g_{\omega 4}}{4 !} V_{0}^{4}+\frac{g_{\sigma \omega}}{4} \phi_{0}^{2} V_{0}^{2} \\
& +\left(\frac{m_{\rho}^{2}}{2}+\frac{g_{\rho 4}}{4 !} R_{0}^{2}+\frac{g_{\sigma \rho}}{4} \phi_{0}^{2}+\frac{g_{\omega \rho}}{4} V_{0}^{2}\right) R_{0}^{2}+\sum_{l=e^{-}, \mu^{-}} \frac{1}{3 \pi^{2}} \int_{0}^{k_{F_{l}}} d k \frac{k^{4}}{E_{l}^{*}(k)^{\prime}}
\end{aligned}
$$

where $k_{F_{B}}$ is the Fermi momentum for baryons. One can check that the thermodynamic relations, such as $\varepsilon_{\mathrm{NHA}}+p_{\mathrm{NHA}}=\rho_{B} E_{n}\left(k_{F_{n}}\right)$ and the chemical potential, $\mu=\partial \varepsilon_{\mathrm{NHA}} / \partial \rho_{B}=$ $E_{n}\left(k_{F_{n}}\right)=E^{*}\left(k_{F_{n}}\right)-\Sigma^{0}\left(k_{F_{n}}\right)$, are exactly satisfied for a given baryon density, $\rho_{B}=2 k_{F}^{3} / 3 \pi^{2}$. Hence, the Hugenholtz-Van Hove theorem to the approximation is exactly maintained in all densities. In Figures 1 and 2, the binding energies of $(n, p, e)-\left(n, p, \Sigma^{-}, e\right)$ and $(n, p, e)-$ $(n, p, \Lambda, e)$ matter are shown. By comparing binding energies of phase transitions from $(n, p, e)$ to $(n, p, H, e)$ matter, it is clearly examined that the equation of state (EOS) becomes softer when a hyperon, $H$, is produced. Note that the bare hyperon-coupling ratios are defined by $r_{\Sigma^{-} N}^{\sigma}=g_{\sigma \Sigma^{-}} / g_{\sigma N}$ and $r_{\Lambda N}^{\sigma}=g_{\sigma \Lambda} / g_{\sigma N}$. The phase transition begins at $k_{F_{\Sigma^{-}}} \sim 1.6 \mathrm{fm}^{-1}$ and $k_{F_{\Lambda}} \sim 1.7 \mathrm{fm}^{-1}$. As one can notice from Figures 1 and 2, the onset densities do not change with the given ratios, $r_{H N}^{\sigma}=1,2 / 3,1 / 3$, expected from effective quark models [26]. Although properties of nuclear matter and EOS of neutron stars are sensitive to nonlinear interactions, the hyperon-onset densities confined by conservation laws and phase-equilibrium conditions 


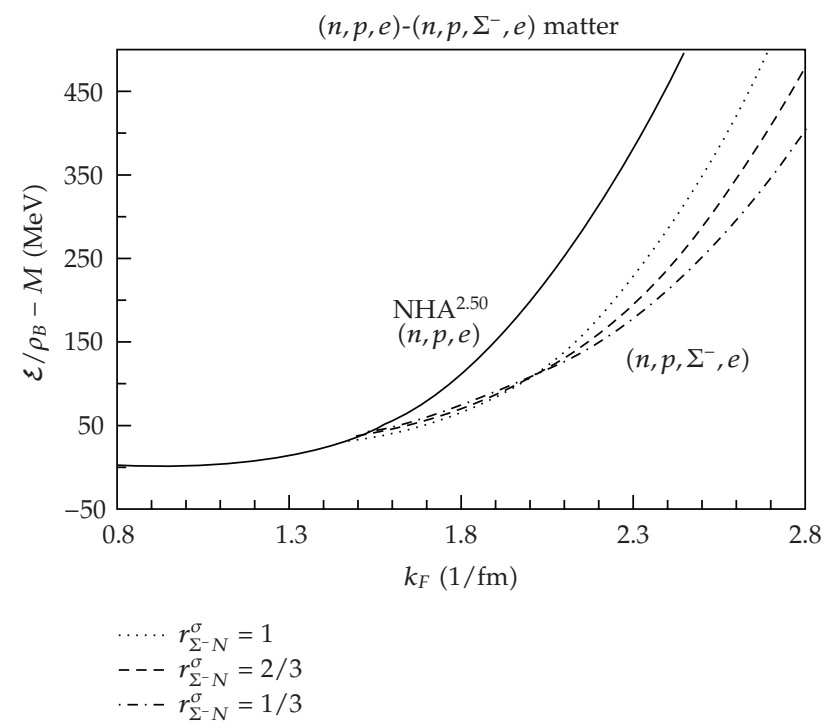

Figure 1: The binding energies of $(n, p, e)$ and $\left(n, p, \Sigma^{-}, e\right)$. The onset density of $\Sigma^{-}$is about $k_{F}=1.6 \mathrm{fm}^{-1}$ as shown in the figure. The ratios of $\Sigma^{-}$-coupling constant on $\sigma$ are (dotted line) $r_{\Sigma^{-} N}^{\sigma}=g_{\sigma \Sigma^{-}} / g_{\sigma N}=1.0$, and (dashed line) $r_{\Sigma^{-} N}^{\sigma}=2 / 3$, (dotted-dashed line) $r_{\Sigma^{-} N}^{\sigma}=1 / 3$, respectively. The other coupling constants are fixed as in Table 1 .

indicate that the hyperon-onset densities are fairly fixed with respect to the change of nonlinear interactions in the current conserving mean-field approximation (see Table 1). The hyperon-onset densities seem to be density-independent, though properties of nuclear and neutron matter are strongly density-dependent.

The equations of motion, self-energies (2.6) and (2.9) enable one to obtain the effective coupling constants and masses, (2.1) and (2.2). In Figures 3 and 4, the effective masses of nucleons and hyperons $\left(\Sigma^{-}, \Lambda\right)$ after hyperon-onset densities are shown, respectively. The hyperon effective masses, $M_{\Sigma^{-}}^{*}$ and $M_{\Lambda^{*}}^{*}$, behave almost the same as those of nucleons in high densities when the hyperon coupling ratios are $r_{H N}^{\sigma}=1$. However, the other values of ratios, $r_{H N}^{\sigma}=1 / 3,2 / 3$, indicate that density dependence of hyperons to effective masses are small in high densities and generate softer EOS, resulting in the lower maximum masses of neutron stars (see Table 1 ). As the softer EOS is examined in the two-fold hyperon matter, $\left(n, p, \Sigma^{-}, \Lambda, e\right)$, it may be conjectured that many-hyperon matter $\left(n, p, \Sigma^{-}, \Lambda, H_{1}, H_{2}, \ldots, e\right)$ with the ratio, $r_{H N}^{\sigma}<1$, would generate much softer EOS and be unable to support observed masses of neutron stars. In addition, many studies with hadronic field theory model independently indicate strong density-dependent interactions and correlations among properties of nuclear matter and neutron stars. Hence, the coupling ratios, $r_{H N}^{\sigma}<1$, predicted by quark-based effective models may not be compatible with those of hadronic models, which should be rigorously investigated to examine consistency and restriction of both hadronic and effective quark models.

\section{The Phase Transition Conditions and Hyperon-Onset Densities}

The hyperon-onset densities at phase transition are given by chemical potentials as

$$
\mu_{H}=\mu_{n}-q_{H} \mu_{e}
$$




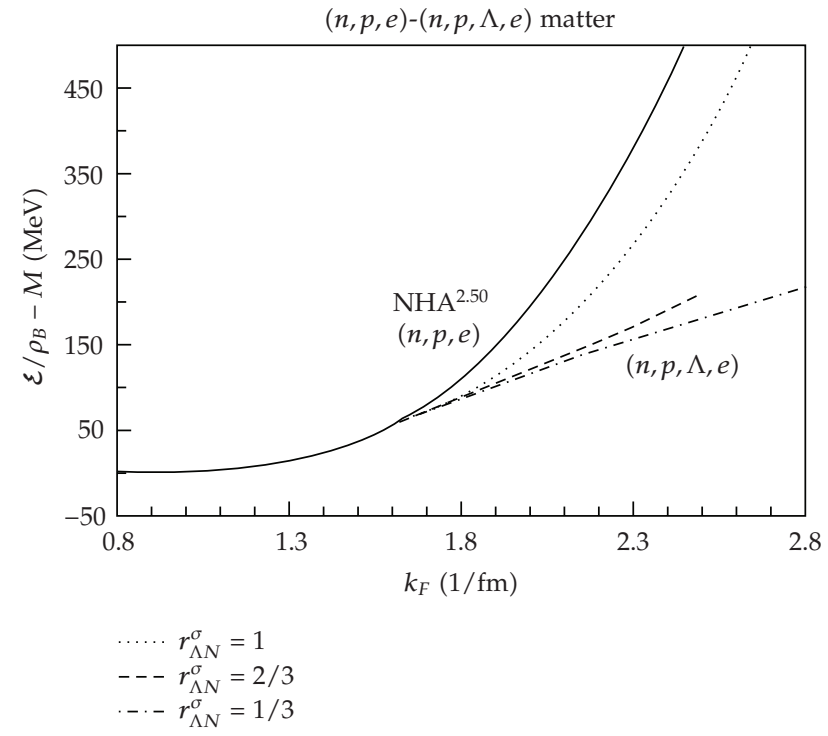

Figure 2: The binding energies of $(n, p, e)$ and $(n, p, \Lambda, e)$. The onset density of $\Lambda$ is about $k_{F}=1.7 \mathrm{fm}^{-1}$ as shown in the figure. The ratios of $\Lambda$-coupling constant on $\sigma$ are (dotted line) $r_{\Lambda N}^{\sigma}=g_{\sigma \Lambda} / g_{\sigma N}=1.0$, and (dashed line) $r_{\Lambda N}^{\sigma}=2 / 3$, (dotted-dashed line) $r_{\Lambda N}^{\sigma}=1 / 3$, respectively. The other coupling constants are fixed as in Table 1 .

where $\mu_{H}, \mu_{n}$, and $\mu_{e}$, are the hyperon, neutron, and electron chemical potentials, and $q_{H}$ is the hyperon charge in the unit of $e$. The phase transition conditions (3.1) are generally obtained by minimizing the energy density $\mathcal{\varepsilon}(n, p, H, e)$, and the baryons are restricted by the baryon-number conservation and charge neutrality. The leptons are produced to maintain charge neutrality, and the lepton densities slowly increase for a low density region, but they decrease rapidly and vanish in high densities since the energies of leptons are absorbed and used to produce higher energy hyperons. The muon can be generated but restricted in a region narrower than that of an electron with the phase-equilibrium condition, $\mu_{\mu^{-}}=\mu_{e^{-}}$, and so, the effect of the muon chemical potential is smaller than that of an electron.

The hyperon-onset densities are determined by chemical potentials which are equal to the single particle energy. The single particle energies of baryons, $E_{B}(k)=\left(k^{2}+\right.$ $\left.M_{B}^{* 2}\right)^{1 / 2}-\Sigma_{\omega, \rho}^{0}\left(k_{B}\right)$, are related to self-energies which depend on effective masses and coupling constants induced by nonlinear interactions. The phase-equilibrium conditions (3.1) are complicated equations which interrelate the density-dependent interactions with hyperononset densities. The hyperon-onset densities are important to determine the maximum masses of neutron stars, since the generation of hyperons will soften the EOS of hyperonmixed nuclear matter. The EOS of hyperons depends also on binding energy and hyperon coupling constants given by density-dependent effective masses and coupling constants of nucleons. In this way, the correlations between properties of nuclear matter and hyperonic matter are intimately constructed to each other. The coupling constants of hyperons, $r_{H N}^{\sigma}$ and $r_{H N}^{\omega}$, play an essential role to determine onset densities.

The hyperon coupling constants, $r_{H N}^{\omega}$ can be calculated in terms of the effective masses, coupling constants, and binding energies of hyperons in the current conserving mean-field approximation. For example, suppose that $(n, p, H, e)$ phase is generated after $(n, p, e)$ phase. The hyperon-onset density is determined by the phase transition conditions 


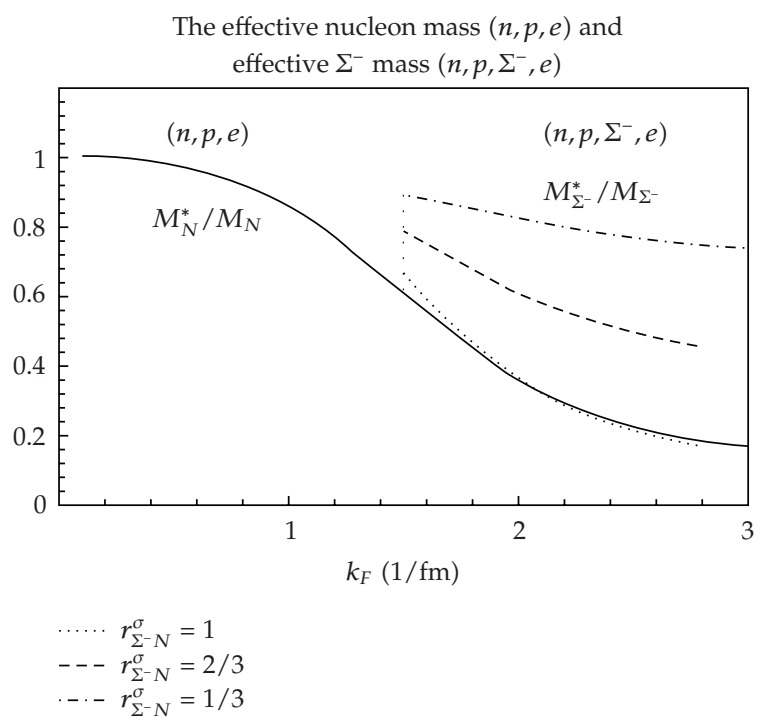

Figure 3: The effective masses of $N$ and $\Sigma^{-}$. Note that the effective mass of hyperon shows $M_{\Sigma^{-}}^{*} / M_{\Sigma^{-}} \sim 1$ as $r_{\Sigma^{-} N}^{\sigma}=1 / 3$. The smaller coupling ratios mean less density-dependent interactions for the hyperon.

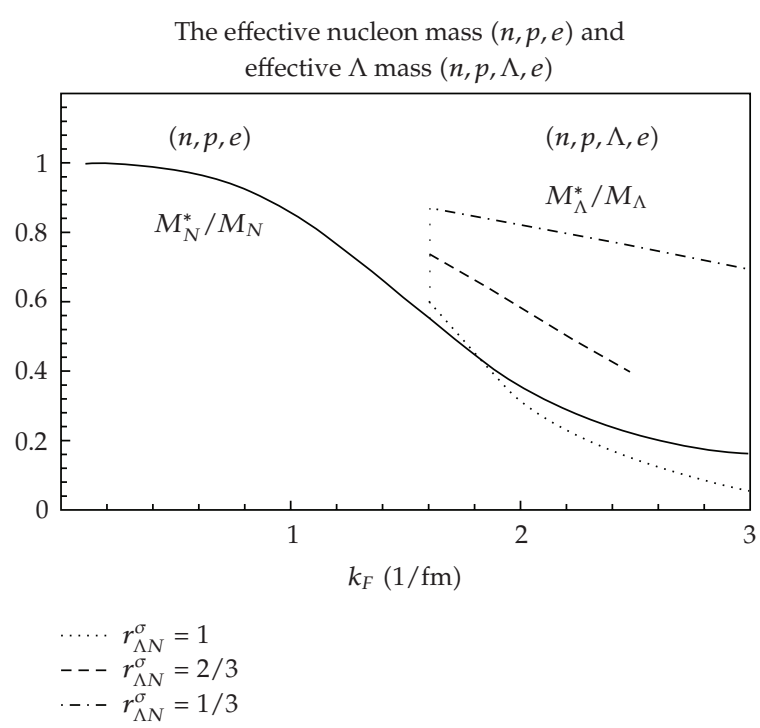

Figure 4: The effective masses of $N$ and $\Lambda$. Note that the effective mass of hyperon shows $M_{\Lambda}^{*} / M_{\Lambda} \sim 1$ as $r_{\Lambda N}^{\sigma}=1 / 3$. The smaller coupling ratios indicate less density-dependent interactions for the hyperon.

(3.1), and the binding energy at the onset-density, $\alpha_{H}$, should be the lowest energy level of the hyperon $H$ (the hyperon single particle energy at saturation). The Hugenholtz-Van Hove theorem of a self-bound system at the onset density $\left(\rho_{H}=0\right)$ leads to

$$
\begin{aligned}
\alpha_{H}=\left(\left(\frac{\varepsilon}{\rho_{B}}\right)_{H}-M_{H}\right)_{\rho_{H}=0} & =E_{H}(0)-M_{H}=E_{H}^{*}(0)-\Sigma_{\omega H}^{0}-M_{H} \\
& =g_{\omega H} V_{0}+M_{H}^{*}-M_{H} .
\end{aligned}
$$


Table 1: Properties of nuclear matter and $\left(n, p, \Sigma^{-}, e\right),(n, p, \Lambda, e)$ neutron stars. The properties of symmetric nuclear matter connected with isospin-asymmetric, beta-equilibrium matter $(n, p, e)$ whose EOS produces $M_{\max }(n, p, e)=2.50 M_{\odot}$ are listed. The coupling constants are chosen from the data $\mathrm{NHA}^{2.50}$ in the paper $[20,21]$.

\begin{tabular}{|c|c|c|c|c|c|c|c|c|c|}
\hline $\begin{array}{l}g_{\sigma} \\
9.326\end{array}$ & $\begin{array}{c}g_{\omega} \\
10.421\end{array}$ & $\begin{array}{c}g_{\rho} \\
4.765\end{array}$ & $\begin{array}{c}g_{\sigma 3}(\mathrm{MeV}) \\
10.0\end{array}$ & $\begin{array}{c}g_{\sigma 4} \\
20.0\end{array}$ & $\begin{array}{l}g_{\omega 4} \\
20.0\end{array}$ & $\begin{array}{c}g_{\rho 4} \\
4.00\end{array}$ & $\begin{array}{l}g_{\sigma \omega} \\
18.0\end{array}$ & $\begin{array}{c}g_{\sigma \rho} \\
-18.0\end{array}$ & $\begin{array}{c}g_{\omega \rho} \\
-18.0\end{array}$ \\
\hline$g_{\sigma \sigma N}$ & $g_{\sigma \omega N}$ & $g_{\sigma \rho N}$ & $g_{\sigma}^{*}$ & $g_{\omega}^{*}$ & $g_{\rho}^{*}$ & & & & \\
\hline-0.018 & 0.013 & 0.048 & 9.063 & 10.800 & 7.567 & & & & \\
\hline $\begin{array}{l}M_{N}^{*} / M \\
0.70\end{array}$ & $\begin{array}{c}m_{\sigma}^{*} / m_{\sigma} \\
1.02\end{array}$ & $\begin{array}{c}m_{\omega}^{*} / m_{\omega} \\
1.01\end{array}$ & $\begin{array}{c}K(\mathrm{MeV}) \\
329\end{array}$ & $\begin{array}{c}a_{4}(\mathrm{MeV}) \\
30.0\end{array}$ & & & & & \\
\hline
\end{tabular}

Table 2: The maximum masses, $M_{\max }$, and central energy densities, $\varepsilon_{C}\left(10^{15} \mathrm{~g} / \mathrm{cm}^{3}\right)$, of neutron stars produced by way of (a) $(n, p, e)-\left(n, p, \Sigma^{-}, e\right)$ and (b) $(n, p, e)-(n, p, \Lambda, e)$ are listed, respectively, with the same coupling constants. The EOS of the hyperon phase $\left(n, p, \Sigma^{-}, e\right)$ and $(n, p, \Lambda, e)$ is calculated with the ratio $r_{H N}^{\sigma}=g_{\sigma H} / g_{\sigma N}=1.0,2 / 3,1 / 3[26]$.

(a)

\begin{tabular}{lcc}
\hline & $(n, p, e)-\left(n, p, \Sigma^{-}, e\right)$ & \\
\hline$r_{\Sigma^{-} N}^{\sigma}$ & $M_{\max }$ & $\mathcal{E}_{c}$ \\
\hline 1.00 & 2.22 & 2.14 \\
$2 / 3$ & 2.08 & 2.24 \\
$1 / 3$ & 1.93 & 1.21 \\
\hline
\end{tabular}

(b)

\begin{tabular}{lcc}
\hline & $(n, p, e)-(n, p, \Lambda, e)$ & \\
\hline$r_{\Lambda N}^{\sigma}$ & $M_{\max }$ & $\varepsilon_{c}$ \\
\hline 1.00 & 2.22 & 2.15 \\
$2 / 3$ & 1.67 & 1.00 \\
$1 / 3$ & 1.56 & 1.02 \\
\hline
\end{tabular}

By employing the effective masses of baryons (2.5) and the self-energy of $\omega$-meson (2.9) with $\Sigma_{\omega}^{0}=-g_{\omega N}^{*} V_{0}$, one can obtain

$$
r_{H N}^{\omega}=\frac{m_{\omega}^{* 2}}{g_{\omega N} g_{\omega N}^{*} \rho_{\omega}}\left(\frac{g_{\sigma H}}{g_{\sigma N}^{*}}\left(M_{N}-M_{N}^{*}\right)+\alpha_{H}\right)=\frac{m_{\omega}^{* 2}}{g_{\omega N} g_{\omega N}^{*} \rho_{\omega}}\left(M_{H}-M_{H}^{*}+\alpha_{H}\right),
$$

where $\rho_{\omega}=\rho_{p}+\rho_{n}$, since $\rho_{H}=0 ; \alpha_{H}$ is the lowest binding energy of isospin symmetric hyperon matter. The hyperon-nucleon coupling ratio is determined by the density-dependent ratio, $g_{\sigma H} / g_{\sigma N}^{*}$. Hence, the hyperon-coupling constants and the lowest binding energies of hyperons are constrained with effective coupling constants, masses of hadrons, nonlinear interactions, nuclear observables, and masses of neutron stars. The hyperon-onset density and hyperon EOS are intimately related to nonlinear interactions and properties of nuclear matter.

With a given ratio $r_{H N^{\prime}}^{\sigma}$, the hyperon-onset densities are calculated by the phaseequilibrium conditions (3.1) and the hyperon-nucleon coupling ratio $r_{H N}^{\omega}$ (3.3), which are complicated functions of single particle energy and self-energies, effective masses, and coupling constants of hadrons. Figures 5 and 6 show the EOS of $(n, p, e)-\left(n, p, \Sigma^{-}, e\right)$ and 


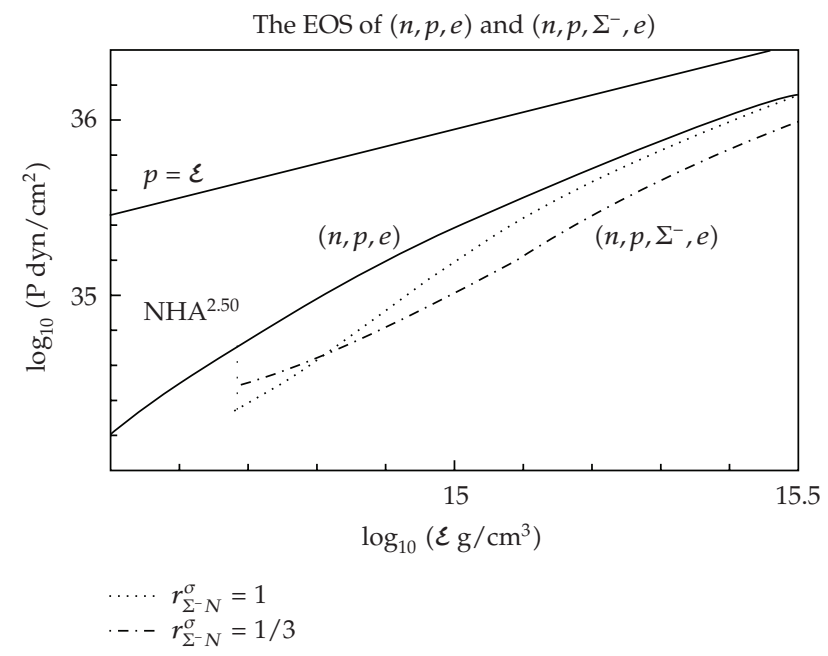

Figure 5: The equation of state for $(n, p, e)-\left(n, p, \Sigma^{-}, e\right)$. Note that the equation of state for $r_{\Sigma^{-} N}^{\sigma}=1 / 3$ becomes softer in $\left(n, p, \Sigma^{-}, e\right)$ phase.

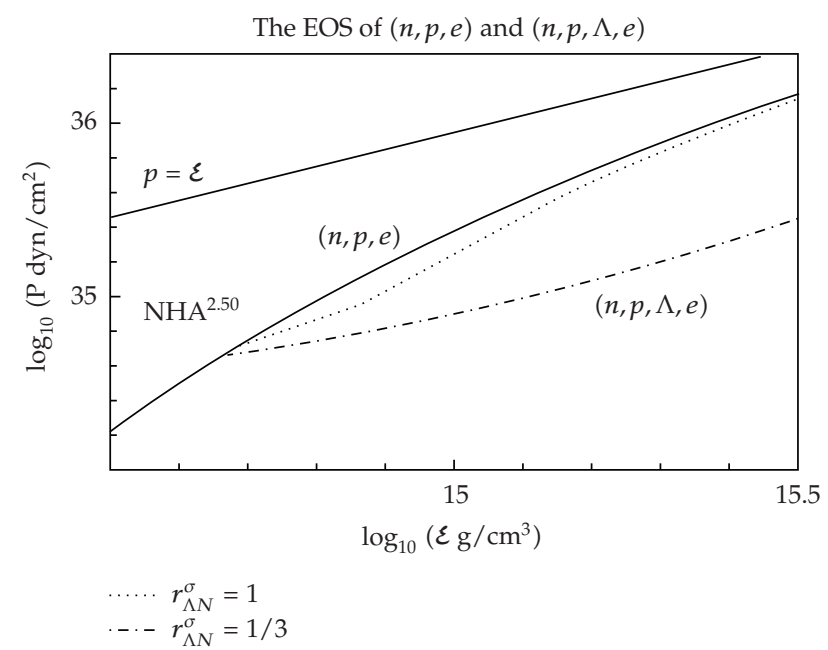

Figure 6: The equation of state for $\Lambda$ from $(n, p, e)$ to $(n, p, \Lambda, e)$. The EOS for $r_{\Lambda N}^{\sigma}=1 / 3$ becomes softer in $(n, p, \Lambda, e)$ phase.

$(n, p, e)-(n, p, \Lambda, e)$ matter with coupling ratios on $\sigma, r_{H N}^{\sigma}=1.0,(1 / 3)\left(r_{H N}^{\sigma}=2 / 3\right.$ is omitted to be concise). The EOSs after hyperon-onset densities become softer in the density range important to determine the masses of neutron stars. In addition, it can be checked numerically that the $\Lambda$-onset density, $k_{F_{\Lambda}}$ in the two-fold hyperon production such as $\left(n, p, \Sigma^{-}, \Lambda, e\right)$, for example, is different from that of $(n, p, \Lambda, e)$. The $\Lambda$-onset density in $\left(n, p, \Sigma^{-}, \Lambda, e\right)$ is pushed up to a high density: $k_{F_{\Lambda}} \sim 2.2 \mathrm{fm}^{-1}$. This holds in general for other hyperons, since the additional new hyperon production requires high energy and pressure for nucleons to absorb energies of leptons so that nucleons can transform to hyperons. The hyperon phase, $\left(n, p, \Sigma^{-}, \Lambda, e\right)$, exists in the density range relevant to determine properties of neutron stars, and furthermore, the EOS is again softened by $\Lambda$ production. Hence, the $\beta$-equilibrium matter, 
$(n, p, e)$, and the hyperon matter, $\left(n, p, \Sigma^{-}, e\right),\left(n, p, \Sigma^{-}, \Lambda, e\right)$, would be more important than $\left(n, p, \Sigma^{-}, \Lambda, H_{1}, H_{2}, \ldots, e\right)$ in order to study the maximum masses of stable neutron stars and properties of nuclear matter.

\section{Incompressibilities and Symmetry Energies for High Density}

The equation of state (EOS) given by $\left(\varepsilon_{\mathrm{NHA}}, p_{\mathrm{NHA}}, \rho_{B}\right)$ and Tolman -Oppenheimer-Volkoff (TOV) equation [27-29] will enable one to calculate properties of nuclear matter at saturation and neutron stars. The values of nonlinear coupling constants are adjusted so that the binding energy at saturation is $-15.75 \mathrm{MeV}$ at $k_{F}=1.30 \mathrm{fm}^{-1}$, and the symmetry energy is $a_{4}=30.0 \mathrm{MeV}$, searching simultaneously the lower bound of nuclear incompressibility, $K$, which corresponds to the maximum mass of neutron stars. The results are listed in Table 1. The coupling constants and effective masses, nonlinear interactions are strictly confined with these imposed constraints, and consequently, physical quantities exhibit strong density-dependent correlations. The derivation of equation of state, incompressibility and symmetry energy, correlations among properties of nuclear and neutron matter in the conserving nonlinear mean-field approximation have been discussed in detail [20,21]. We exhibit characteristic density-dependent correlations of properties of nuclear matter such as incompressibility and symmetry energy of $(n, p, e)-\left(n, p, \Sigma^{-}, e\right)$ and $(n, p, e)-(n, p, \Lambda, e)$ matter.

The incompressibility, $K$, and nucleon symmetry energy, $a_{4}$, are respectively, calculated in the conserving mean-field approximation as $[30,31]$

$$
K=9 \rho_{B} \frac{\partial^{2} \varepsilon}{\partial \rho_{B}^{2}}, \quad a_{4}=\frac{1}{2} \rho_{N}\left[\left[\frac{\partial^{2} \varepsilon}{\partial \rho_{3}^{2}}\right]_{\rho_{N}}\right]_{\rho_{3}=0} .
$$

The computation of nucleon symmetry energy must be performed by maintaining phaseequilibrium conditions, which will fix mean-fields, $\phi_{0}, V_{0}$, and $R_{0}$ and the ground state energy, $\mathcal{E}\left(\rho_{p}, \rho_{n}\right)$; then, the derivative of the energy density $\mathcal{\varepsilon}\left(\rho_{p}, \rho_{n}\right)$ can be calculated by changing $\rho_{p}$ and $\rho_{n}$ with fixed $\rho_{N}=\rho_{p}+\rho_{n}$ and mean fields. The hyperon onset and softening of EOS are perceived as the discontinuity and abrupt reduction of incompressibility shown in Figure 7. This characteristic property can be understood from the decreasing slope of binding energy curves in Figures 1 and 2 and would significantly change incompressibility, symmetry energy, and Landau parameters in high densities, which should be examined, for example, in heavy-ion collision experiments as a signal for the hyperon production [30-33]. The symmetry energies are monotonically increasing around saturation density, while they saturate in high densities [20,21], as shown in Figure 8; the saturation of symmetry energy in a high density is also numerically checked in hyperon matter. The theoretical calculations of $K$ and $a_{4}$ depend on interactions of baryons, many-body interactions, and constraints such as isospin asymmetry and charge neutrality. The current results are different in high densities from those discussed in articles [30-35].

The lowest binding energies of $\Lambda$ and $\Sigma^{-}$are fixed in the current calculation as $-28 \mathrm{MeV}$ and $20 \mathrm{MeV}$, respectively, [26,36-38]. The lowest binding energies are related to the hyperon-coupling strength as shown in (3.3). We have checked the hyperon-onset densities by changing the values of binding energies to examine whether onset densities can be noticeably changed or not. In the numerical analysis, the hyperon-onset densities are fairly 


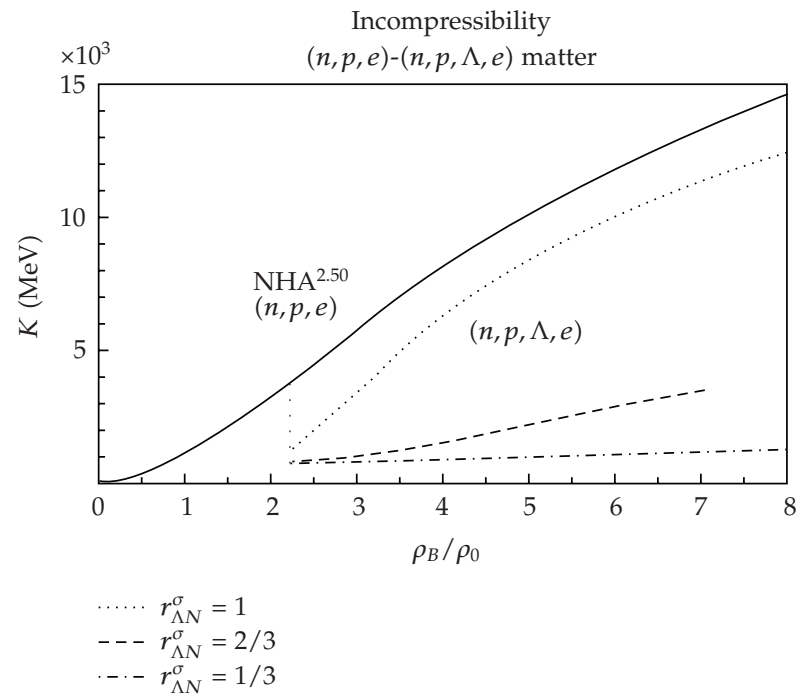

Figure 7: Incompressibilities of $(n, p, e)$ and $(n, p, \Lambda, e)$. The discontinuity of incompressibility occurs at $k_{F}=1.7 \mathrm{fm}^{-1}$, because of the phase transition from $(n, p, e)$ to $(n, p, \Lambda, e) . \rho_{0}$ is the saturation density of symmetric nuclear matter.

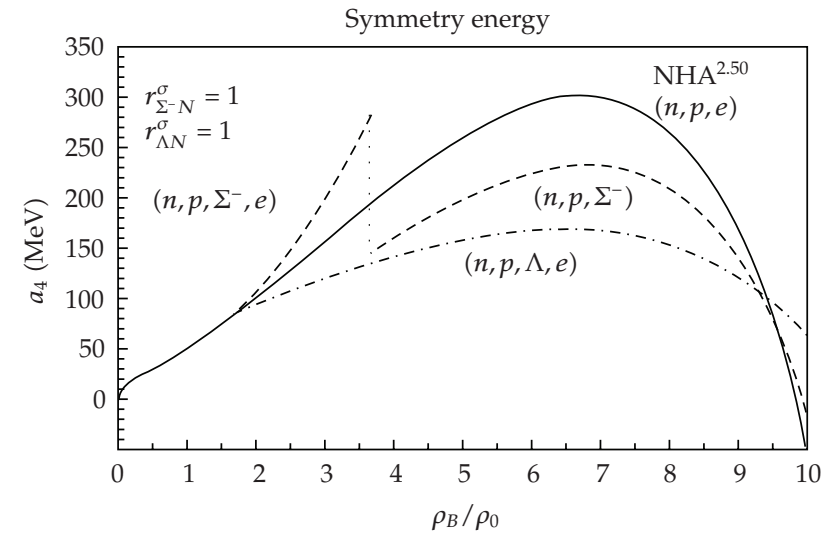

Figure 8: Nucleon symmetry energies of $(n, p, e),\left(n, p, \Sigma^{-}, e\right)$, and $(n, p, \Lambda, e)$ are shown with the solid, doted, and dot-dashed lines, respectively. The ratios of hyperon coupling constants are $r_{\Sigma^{-} N}^{\sigma}=1.0$ and $r_{\Lambda N}^{\sigma}=1.0$. The discontinuity of symmetry energy, $\left(n, p, \Sigma^{-}, e\right)$, occurs at $\rho_{B} / \rho_{0} \sim 3.641$, because of the phase transition from $\left(n, p, \Sigma^{-}, e\right)$ to $\left(n, p, \Sigma^{-}\right)$.

fixed with changes of $\alpha_{H}$, if $\left|\alpha_{H}\right|$ is confined smaller than $g_{\sigma H} / g_{\sigma N}^{*}\left(M_{N}-M_{N}^{*}\right)$; experimental values of $\left|\alpha_{H}\right|$ are typically smaller than $g_{\sigma H} / g_{\sigma N}^{*}\left(M_{N}-M_{N}^{*}\right)$. It suggests that effective masses and coupling constants be more important to determine hyperon-onset densities than binding energies are. However, the binding energies of hyperons $\alpha_{H}$, effective masses, and coupling constants are important factors to determine the EOS and properties for neutron stars. Therefore, hyperon-onset densities, binding energies, and nonlinear interactions of hadrons will intimately interrelate properties of nuclear matter with those of neutron stars. 


\section{Remarks}

The current conserving mean-field approximation and renormalized nonlinear interactions have exhibited interesting density-dependent correlations among observables of nuclear and high-density hyperonic matter.

(1) The hyperon-onset densities are fairly fixed, respectively, although densitydependent interactions prominently affect the EOS and properties of nuclear and neutron matter. Therefore, the hyperon-onset density could be one of the important constraints on theoretical and experimental models of high density, exotic nuclear matter. The signals of hyperon production and onset density should be investigated further in heavy-ion collision experiments, and the results obtained in the current investigation should be examined carefully for nonlinear interactions including all other hyperons.

(2) The onset density of $\Lambda$ in the two-fold hyperon phase, $\left(n, p, \Sigma^{-}, \Lambda, e\right)$, shifts to a higher density than that of $(n, p, \Lambda, e)$, and the EOS becomes softer. The two-fold hyperon production requires high energy and pressure restricted by phase-equilibrium conditions, and Fermi energies of baryons will be redistributed among baryons to maintain the phase-equilibrium conditions and constraints, resulting in the reduction of Fermi energies (chemical potentials). The chemical potentials of leptons tend to be converted to those of baryons in high densities, and leptons vanish so that nuclear matter become baryons-only phase (e.g., $\left(n, p, \Sigma^{-}, e\right)-\left(n, p, \Sigma^{-}\right)$phase transition in Figure 8$)$. The conversion of chemical potentials among baryons in order to satisfy phase-transition conditions and constraints can be observed numerically with newly generated hyperons. The characteristic feature increases the hyperon-onset density higher and makes the EOS softer. Hence, it suggests that properties of neutron stars be mainly determined by $(n, p, e),\left(n, p, \Sigma^{-}, e\right)$, and $\left(n, p, \Sigma^{-}, \Lambda, e\right)$ matter rather than $\left(n, p, \Sigma^{-}, \Lambda, H_{1}, H_{2}, \ldots, e\right)$ matter; many-hyperon matter could be possible in a high density, such as in the core of neutron stars.

(3) The softening of EOS and discontinuity of incompressibility are interrelated to the strength of the hyperon coupling constants and effective masses of mesons and hyperons; hence, theoretical and experimental analyses of incompressibility and EOS in high densities are essential to determine physical quantities. The discontinuous change is also obtained for the symmetry energy for $(n, p, e)-\left(n, p, \Sigma^{-}, e\right)-\left(n, p, \Sigma^{-}\right)$matter. The symmetry energy is monotonically increasing in the density range, $\rho_{B} / \rho_{0} \lesssim 3.0$, but it saturates in a high density (see Figure 8); the saturation of symmetry energy is the effect of both nonlinear interactions and isospin asymmetry $[20,21]$. The theoretical predictions for the symmetry energy are very different in high densities. The value should be investigated actively in heavy-ion collision and other experiments to discriminate these predictions [34, 35].

(4) The binding energies, effective masses, and coupling constants of hyperons generate strong density correlations among properties of nuclear matter and neutron stars. Therefore, the binding energies and coupling ratios of hyperons, the hyperon-onset densities and signals of phase transition of $(n, p, e),\left(n, p, \Sigma^{-}, e\right)$, and $\left(n, p, \Sigma^{-}, \Lambda, e\right)$, will, respectively, exhibit important information on saturation properties (the binding energy and density, incompressibility, and symmetry energy) not only for isospin-symmetric but also for isospinasymmetric nuclear matter and neutron stars [39].

(5) The values of hyperon coupling ratios, $\left(r_{\Lambda N}^{\sigma} \sim 1,1 / 3,2 / 3\right)$, yield consistent results with the central energy density and the maximum mass configuration [5]. However, the hyperon coupling ratios, $r_{H N}^{\sigma} \lesssim 1$, suggested by effective quark models indicate that density interactions of baryons are weak in high densities. It seems to be inconsistent with predictions suggested by theoretical models of hadrons that density-dependent interactions be significant 
for nuclear matter and neutron stars. This aspect should be investigated further for both hadronic and effective quark models.

The densities of hyperon onset and phase transitions, $(n, p, e) \rightarrow\left(n, p, \Sigma^{-}, \Lambda, e, \ldots\right)$, are sensitive to coupling ratios given by density-dependent effective masses and coupling constants of nucleons. The hyperon-onset densities and binding energies of hyperons are important to determine properties of EOS and neutron stars. Hence, the consistency of coupling strengths and binding energies of hyperons could be evaluated from certain astronomical data. The results suggest that the analyses of nuclear matter and neutron stars may provide important information on the models of nuclear and astronomical physics. The signals of the abrupt change of EOS, discontinuous change of incompressibility, and the saturation property of symmetry energy are essential to understand high density, exotic nuclear matter. The nonlinear mean-field approximation has exhibited interesting correlations among effective coupling constants and masses of hadrons, incompressibility, symmetry energy, and masses of hyperonic neutron stars. The properties of nuclear matter, neutron stars, and nuclear astrophysics are abundant in interesting physics to one another; the interdisciplinary progresses of these fields would be anticipated in the near future.

\section{Acknowledgments}

The authors would like to acknowledge Professor T. Muto of Chiba Institute of Technology for his valuable comments on binding energies of hyperons. The work is supported by Osaka Gakuin Junior College research grant for the 2008 Academic Year.

\section{References}

[1] B. D. Serot and J. D. Walecka, "The relativistic nuclear many-body problem," in Advances in Nuclear Physics, J. W. Negele and E. Vogt, Eds., vol. 16, Plenum, New York, NY, USA, 1986.

[2] B. D. Serot, "Quantum hadrodynamics," Reports on Progress in Physics, vol. 55, no. 11, pp. 1855-1946, 1992.

[3] N. K. Glendenning, Compact Stars, Springer, New York, NY, USA, 2nd edition, 2000.

[4] A. Akmal, V. R. Pandharipande, and D. G. Ravenhall, "Equation of state of nucleon matter and neutron star structure," Physical Review C, vol. 58, no. 3, pp. 1804-1828, 1998.

[5] J. M. Lattimer and M. Prakash, "Ultimate energy densitinfy of observable cold baryonic matter," Physical Review Letters, vol. 94, no. 11, Article ID 111101, 4 pages, 2005.

[6] F. Weber and M. K. Weigel, "Baryon composition and macroscopic properties of neutron stars," Nuclear Physics A, vol. 505, no. 3-4, pp. 779-822, 1989.

[7] J. Schaffner and I. N. Mishustin, "Hyperon-rich matter in neutron stars," Physical Review C, vol. 53, no. 3, pp. 1416-1429, 1996.

[8] I. Vidaña, A. Polls, A. Ramos, L. Engvik, and M. Hjorth-Jensen, "Hyperon-hyperon interactions and properties of neutron star matter," Physical Review C, vol. 62, no. 3, Article ID 035801, 8 pages, 2000.

[9] J. K. Bunta and Š. Gmuca, "Hyperons in a relativistic mean-field approach to asymmetric nuclear matter," Physical Review C, vol. 70, no. 5, Article ID 054309, 10 pages, 2004.

[10] J. M. Luttinger and J. C. Ward, "Ground-state energy of a many-fermion system. II," Physical Review, vol. 118, no. 5, pp. 1417-1427, 1960.

[11] G. Baym and L. P. Kadanoff, "Conservation laws and correlation functions," Physical Review, vol. 124, no. 2, pp. 287-299, 1961.

[12] G. Baym, "Self-consistent approximations in many-body systems," Physical Review, vol. 127, no. 4, pp. 1391-1401, 1962.

[13] R. J. Furnstahl and B. D. Serot, "Covariant Feynman rules at finite temperature: time-path formulation," Physical Review C, vol. 44, no. 5, pp. 2141-2174, 1991. 
[14] R. J. Furnstahl and B. D. Serot, "Covariant mean-field calculations of finite-temperature nuclear matter," Physical Review C, vol. 41, no. 1, pp. 262-279, 1990.

[15] Y. Takada, "Exact self-energy of the many-body problem from conserving approximations," Physical Review B, vol. 52, no. 17, pp. 12708-12719, 1995.

[16] "Progress in Nonequilibrium Green's Functions I, II," in Proceedings of the Conference "Kadanoff-Baym Equations", M. Bonitz, R. Nareyka, and D. Semkat, Eds., World Scientific, Dresden, Germany, August 2002.

[17] Yu. B. Ivanov, J. Knoll, and D. N. Voskresensky, "Self-consistent approximations to non-equilibrium many-body theory," Nuclear Physics A, vol. 657, no. 4, pp. 413-445, 1999.

[18] Yu. B. Ivanov, J. Knoll, and D. N. Voskresensky, "Resonance transport and kinetic entropy," Nuclear Physics A, vol. 672, no. 1-4, pp. 313-356, 2000.

[19] F. Riek and J. Knoll, "Selfconsistent description of vector-mesons in matter," Nuclear Physics A, vol. 740, no. 3-4, pp. 287-308, 2004.

[20] H. Uechi, "Properties of nuclear and neutron matter in a nonlinear $\sigma-\omega-\rho$ mean-field approximation with self- and mixed-interactions," Nuclear Physics A, vol. 780, no. 3-4, pp. 247-273, 2006.

[21] H. Uechi, "Density-dependent correlations between properties of nuclear matter and neutron stars in a nonlinear $\sigma-\omega$ - $\rho$ mean-field approximation," Nuclear Physics A, vol. 799, no. 1-4, pp. 181-209, 2008.

[22] L. D. Landau, "The theory of a Fermi liquid," Soviet Physics JETP, vol. 3, pp. 920-925, 1956.

[23] L. D. Landau, “Oscillations in a Fermi liquid," Soviet Physics JETP, vol. 5, pp. 101-108, 1957.

[24] N. M. Hugenholtz and L. van Hove, "A theorem on the single particle energy in a Fermi gas with interaction," Physica, vol. 24, no. 1-5, pp. 363-376, 1958.

[25] N. P. Landsman and Ch. G. van Weert, "Real- and imaginary-time field theory at finite temperature and density," Physics Reports, vol. 145, no. 3-4, pp. 141-249, 1987.

[26] J. Mareš, E. Friedman, A. Gal, and B. K. Jenning, "Constraints on $\Sigma$-nucleus dynamics from Dirac phenomenology of $\Sigma$-atoms," Nuclear Physics A, vol. 594, no. 3, pp. 311-324, 1995.

[27] H. Heiselberg and M. Hjorth-Jensen, "Phases of dense matter in neutron stars," Physics Report, vol. 328, no. 5-6, pp. 237-327, 2000.

[28] J. B. Hartle, "Slowly rotating relativistic stars-I: equations of structure," Astrophysical Journal, vol. 150, p. 1005, 1967.

[29] J. B. Hartle, "Slowly-rotating relativistic stars-IV: rotational energy and moment of inertia for stars in differential rotation," Astrophysical Journal, vol. 161, p. 111, 1970.

[30] T. Matsui, "Fermi-liquid properties of nuclear matter in a relativistic mean-field theory," Nuclear Physics A, vol. 370, no. 3, pp. 365-388, 1981.

[31] H. Uechi, "Fermi-liquid properties of nuclear matter in a Dirac-Hartree-Fock approximation," Nuclear Physics A, vol. 501, no. 4, pp. 813-834, 1989.

[32] M. Kutschera and J. Niemiec, "Mixed quark-nucleon phase in neutron stars and nuclear symmetry energy," Physical Review C, vol. 62, no. 2, Article ID 025802, 9 pages, 2000.

[33] B.-A. Li, "High density behaviour of nuclear symmetry energy and high energy heavy-ion collisions," Nuclear Physics A, vol. 708, no. 3-4, pp. 365-390, 2002.

[34] C.-H. Lee, T. T. S. Kuo, G. Q. Li, and G. E. Brown, “Nuclear symmetry energy,” Physical Review C, vol. 57, no. 6, pp. 3488-3491, 1998.

[35] A. E. L. Dieperink, Y. Dewulf, D. Van Neck, M. Waroquier, and V. Rodin, “Nuclear symmetry energy and the neutron skin in neutron-rich nuclei," Physical Review C, vol. 68, no. 6, Article ID 064307, 9 pages, 2003.

[36] A. Reuber, K. Holinde, and J. Speth, "Meson-exchange hyperon-nucleon interactions in free scattering and nuclear matter," Nuclear Physics A, vol. 570, no. 3-4, pp. 543-579, 1994.

[37] J. Dabrowski and J. Rożynek, "The associated $\Sigma$ production and the $\Sigma$-nucleus potential," Acta Physica Polonica B, vol. 35, no. 9, pp. 2303-2312, 2004.

[38] T. Muto, "Kaon-condensed hypernuclei as highly dense self-bound objects," Nuclear Physics A, vol. 804, no. 1-4, pp. 322-348, 2008.

[39] H. Uechi, "Correlations between saturation properties of isospin symmetric and asymmetric nuclear matter in a nonlinear $\sigma-\omega-\rho$ mean-field approximation," Advanced Studies in Theoretical Physics, vol. 2, no. 11, pp. 519-548, 2008. 

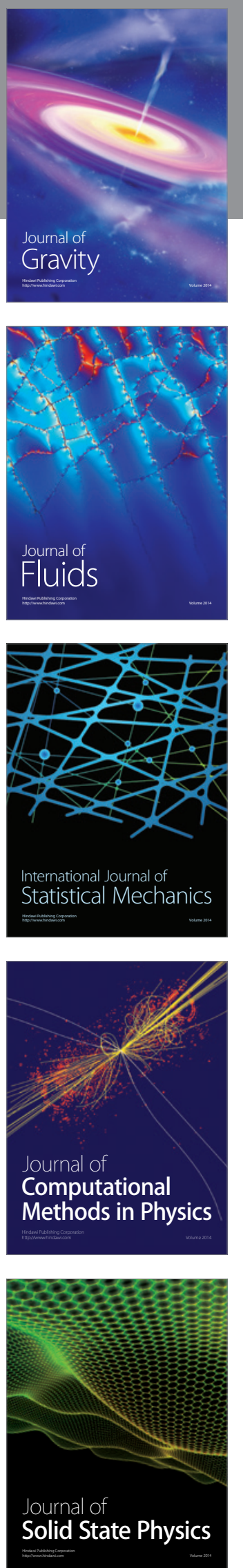

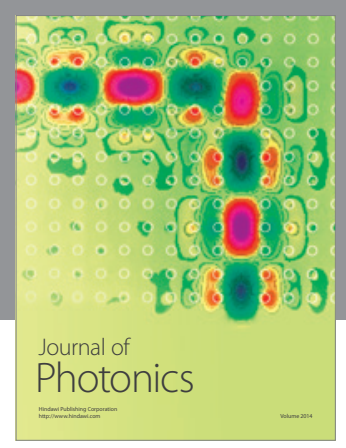

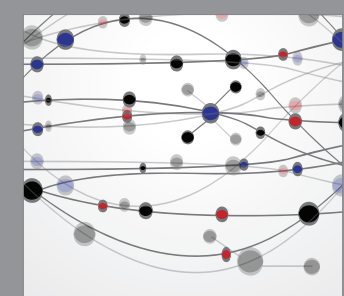

The Scientific World Journal
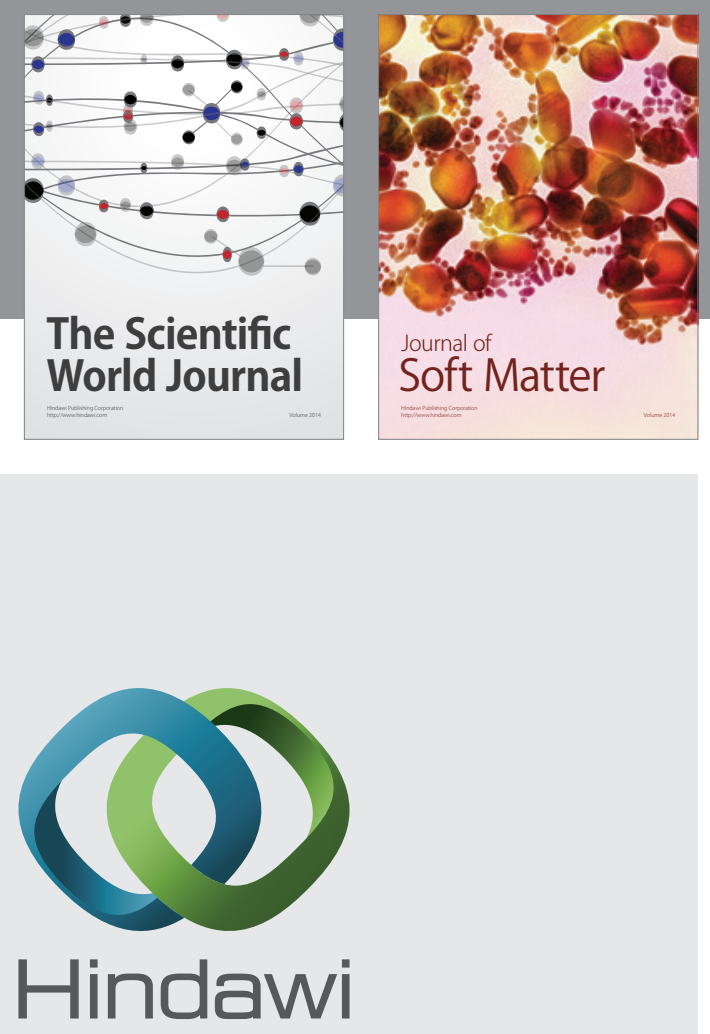

Submit your manuscripts at

http://www.hindawi.com
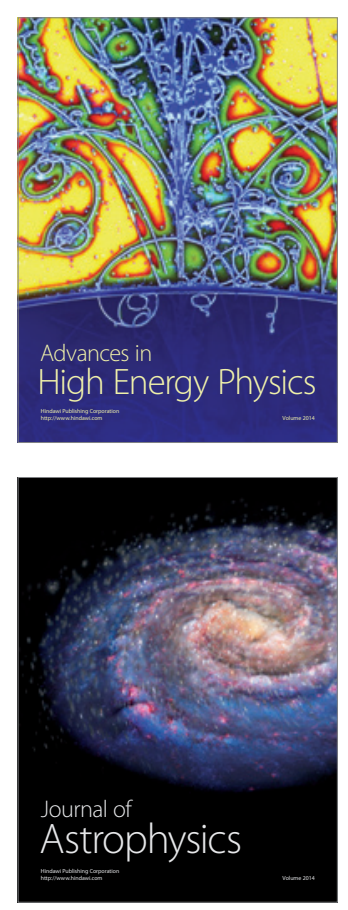
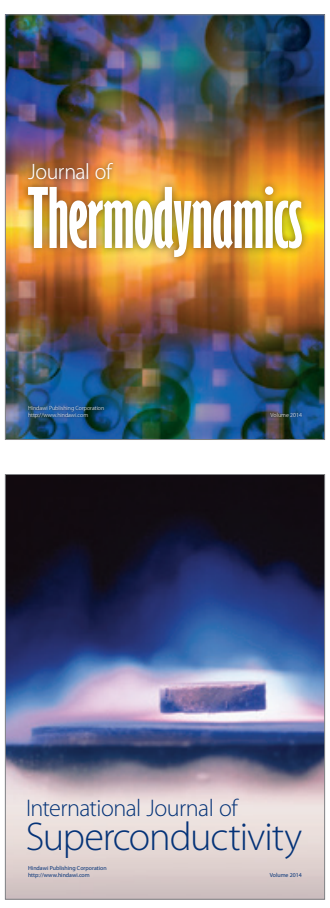
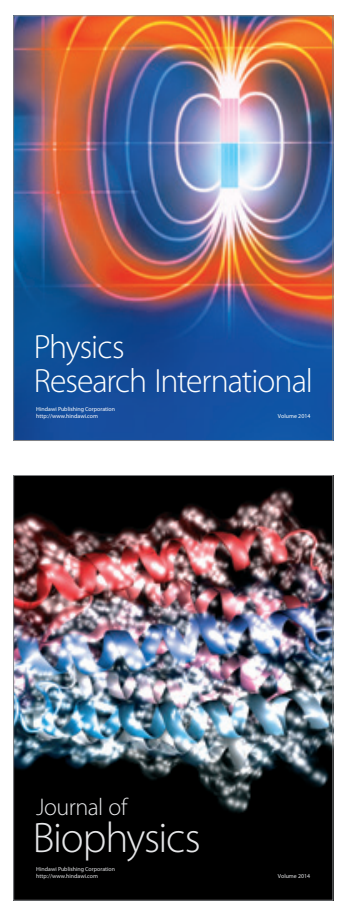
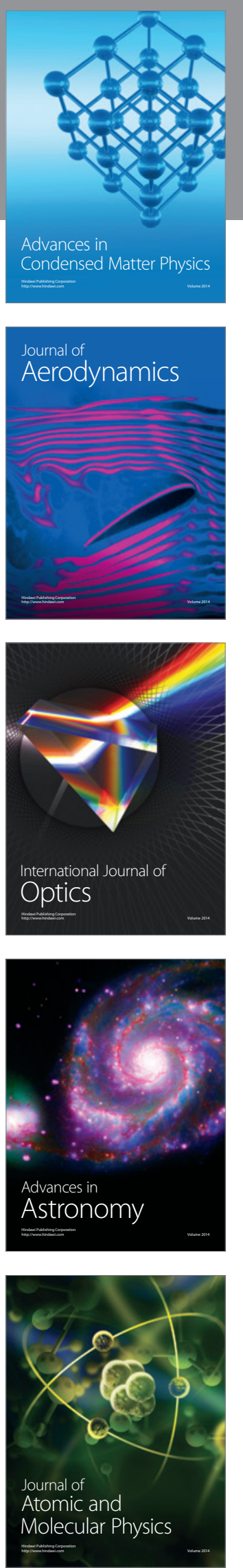